\title{
Dielectric spectroscopy and hydrogen bonding studies of 1-chloropropane-ethanol mixture using TDR technique
}

\author{
Ravindra V. Shinde*, Avadhut R. Deshmukh ${ }^{\dagger}$, Shital A. Ingole ${ }^{\dagger}$ \\ and Ashok C. Kumbharkhane ${ }^{\dagger, \ddagger}$ \\ *College of Agriculture Engineering and Technology \\ Vasantrao Naik Marathwada Krishi Vidyapeeth Basmat Road \\ Parbhani 431402, Maharashtra, India \\ †'School of Physical Sciences \\ Swami Ramanand Teerth Marathwada University \\ Vishnupuri, Nanded 431606, Maharashtra, India \\ †akumbharkhane@yahoo.co.in
}

Received 10 January 2019; Revised 27 March 2019; Accepted 7 April 2019; Published 6 May 2019

\begin{abstract}
The time-domain reflectometry technique was exercised to assess the complex permittivities of 1-chloropropane and ethanol along with their binary mixture in the frequency range of $10 \mathrm{MHz}-50 \mathrm{GHz}$ and in the temperature range of $10-25^{\circ} \mathrm{C}$. Dielectric parameters like static dielectric permittivity $\left(\varepsilon_{0}\right)$ and relaxation time $(\tau)$ have been acquired by the least-square-fit method. The obtained assessments of static permittivity and relaxation time were used to compute various dielectric parameters like excess dielectric constant, excess relaxation time, Kirkwood correlation factor and thermodynamic parameters. The experimental outcome illustrates the nonlinear variations in dielectric permittivity, relaxation time and authenticates the structural formation due to intermolecular interaction between 1-chloropropane and ethanol. Molecular rotary motion and dipole reorientation movements of these complex systems are presented in agreement with the molar entropy and enthalpy.
\end{abstract}

Keywords: Time-domain reflectometry; complex permittivity; dielectric relaxation; thermodynamic parameter; Kirkwood factor.

\section{Introduction}

An exploration to learn the hydrogen bond reorganization dynamics of solvent-solvent as well as pure solvent-mixtures has been extensively made with the help of dielectric relaxation spectroscopy. To identify hydrogen bonding and intermolecular interactions in the mixture, dielectric relaxation studies on binary mixtures play a chief role.

Structurally, 1-chloropropane (CAS No. 540-54-5) is an organochlorine compound as well as a secondary haloalkane, the concluding label recognizes the two $\mathrm{C}-\mathrm{C}$ bonds seen around the carbon atom. 1-Chloropropane is a flammable, colorless liquid with chloroform odor and is prepared by the reaction of $n$-propyl alcohol with hydrochloric acid in the presence of zinc chloride as a catalyst. ${ }^{1}$ It is an organic compound with chemical formula $\mathrm{CH}_{3} \mathrm{CH}_{2} \mathrm{CH}_{2} \mathrm{Cl}$ and a vapor pressure of $345 \mathrm{~mm}-\mathrm{Hg}$ at $25^{\circ} \mathrm{C}$. The inhalation exposure of 1-chloropropane in humans causes central nervous system-related effects characterized by nausea, headache, dizziness, unconsciousness, coma and even respiratory irritation. Also, liquid form of 1-chloropropane can cause eye, skin and digestive irritations. ${ }^{2,3}$ Ethanol $\left(\mathrm{C}_{2} \mathrm{H}_{5} \mathrm{OH}\right)$ is a nonaqueous protic solvent. ${ }^{4}$ It has a dielectric constant $(\varepsilon)$ of 25.07 and a dipole moment $(\mu)$ of $1.70 \mathrm{D} .{ }^{5}$ It is a colorless, inflammable liquid having a low boiling point $\left(78^{\circ} \mathrm{C}\right){ }^{6}$ It is miscible with water in all proportions and is also miscible with most organic solvents. ${ }^{7,8}$ Ethanol is important as an industrial and pharmaceutical solvent.

Numerous researchers have studied the temperaturedependent dielectric relaxation parameters of assorted associating molecule mixtures. Cavity formation and dipolar contribution to the Gauche-trans isomerization of 1-chloropropane and 1,2-dichlroethane have been reported by Melendez-Pagan et al $^{9}{ }^{9}$ Conformational and structural studies of 1-chloropropane and 1-bromopropane from temperaturedependent FT-IR spectra of rare gas solutions and $a b$ initio calculations are reported by Durig et al. ${ }^{10}$

In the present investigation, the dielectric relaxation of 1-chloropropane in ethanol solution at different temperatures $\left(10^{\circ} \mathrm{C}, 15^{\circ} \mathrm{C}, 20^{\circ} \mathrm{C}\right.$ and $\left.25^{\circ} \mathrm{C}\right)$ is undertaken. A regular microwave technique and the solution methods have been used for this study. Dielectric relaxation of polar liquids in polar solvents by microwave absorption study gives valuable information about various types of molecular associations present in the solutions. It is because microwaves can detect weak molecular interactions. ${ }^{11-15}$ A solute-solvent type of molecular association has been proposed. This aspect motivated us to undertake an extensive study to understand the associative nature of 1-chloropropane in polar ethanol

This is an Open Access article published by World Scientific Publishing Company. It is distributed under the terms of the Creative Commons Attribution 4.0 (CC-BY) License. Further distribution of this work is permitted, provided the original work is properly cited. 
environment. The study is projected to present a better perception of the nature of molecular involvement in the mixture.

\section{Experimental}

\subsection{Materials}

1-Chloropropane $\left(\mathrm{CH}_{3} \mathrm{CH}_{2} \mathrm{CH}_{2} \mathrm{Cl}\right.$; CAS No. 540-54-5; purity $98 \%)$ and ethanol $\left(\mathrm{C}_{2} \mathrm{H}_{5} \mathrm{OH}\right.$; purity $\left.99.9 \%\right)$ were purchased from Merck Life Sciences Pvt. Ltd., Mumbai, and used without purification.

\subsection{Measurements}

The complex permittivity of the solution was measured in the frequency range of $10 \mathrm{MHz}-50 \mathrm{GHz}$ at $10-25^{\circ} \mathrm{C}$ using timedomain reflectometry (TDR) technique. ${ }^{16,17}$ The temperature of the samples under test has been maintained using a calibrated temperature controller system with an accuracy of $\pm 0.1^{\circ} \mathrm{C}$. The Tektronix DSA8300 sampling mainframe oscilloscope with dual-channel sampling module $80 \mathrm{E} 10 \mathrm{~B}$ has been used. To feed the pulse, a coaxial cable having $50-\Omega$ impedance, an inner diameter of $0.28 \mathrm{~mm}$ and an outer diameter of $1.19 \mathrm{~mm}$ is used. Sampling oscilloscope monitors changes in pulse after reflection from the end of line. Reflected pulses without sample $R_{1}(t)$ and with sample $R_{x}(t)$ were recorded in a time window of $0.5 \mathrm{~ns}$ and digitized in 2000 points. To determine the complex permittivity spectra $\varepsilon^{*}(\omega)$, the Fourier transformations of the pulse and data analysis were performed using nonlinear least-square-fit method, ${ }^{18,19}$ with $50-\mathrm{GHz}$ bandwidth and TDR module with step generator unit was used.

\section{Results and Discussion}

\subsection{Complex permittivity}

The complex permittivity spectra for different concentrations of 1-chloropropane in ethanol at $25^{\circ} \mathrm{C}$ are shown in Fig. 1. To calculate the static dielectric constant $\left(\varepsilon_{0}\right)$, relaxation time $(\tau)$ and distribution parameters $(\alpha$ and $\beta)$, complex permittivity $\varepsilon^{*}(\omega)$ data were fitted by the nonlinear least-square-fit method to the Havriliak-Negami expression, ${ }^{20}$

$$
\varepsilon^{*}(\omega)=\varepsilon_{\infty}+\frac{\varepsilon_{0}-\varepsilon_{\infty}}{\left[1+(j \omega \tau)^{1-\alpha}\right]^{\beta}},
$$

where $\varepsilon_{0}, \varepsilon_{\infty}, \tau, \alpha$ and $\beta$ are the fitting parameters. The Havriliak-Negami expression includes the Debye $(\alpha=0$, $\beta=1),{ }^{21}$ Cole-Cole $(\beta=1)^{22}$ and Cole-Davidson $(\alpha=0)^{23}$ and relaxation spectral functions in limiting form.

In this investigation, the binary mixture of 1-chloropropane and ethanol confirms Debye-type dispersion. So, the complex permittivity spectra have been fitted in Debyetype model using the nonlinear least-square-fit method to determine the dielectric relaxation parameters. The static dielectric constant and relaxation time for the binary mixture of 1-chloropropane and ethanol at $25^{\circ} \mathrm{C}$ reported in Table 1 agree well with the standards reported in literature.

In the binary liquid systems such as 1-chloropropaneethanol mixture, it has been observed that static dielectric constant $\varepsilon_{0}$ and relaxation time $\left(\tau_{0}\right)$ decrease with the

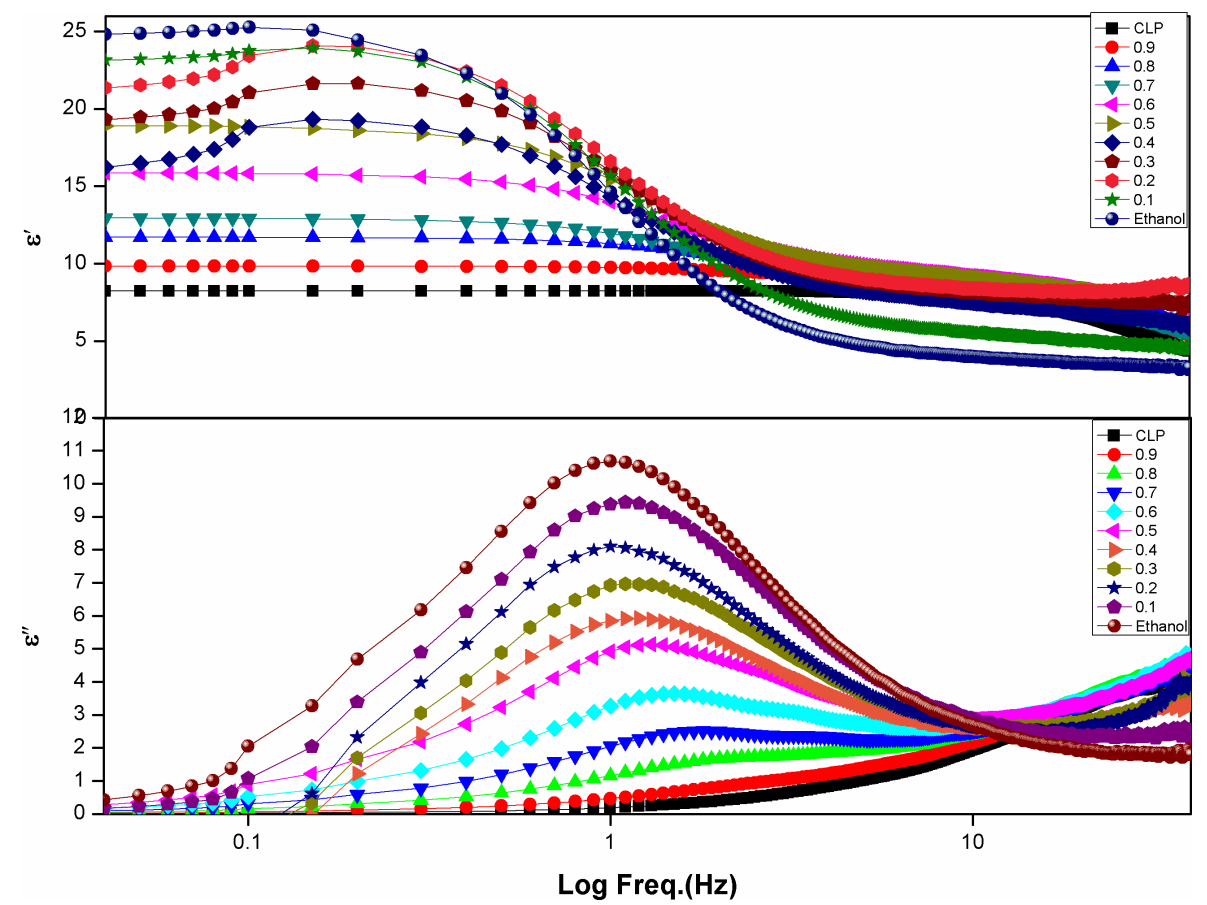

Fig. 1. Frequency-dependent complex dielectric permittivity spectra of 1-chloropropane-ethanol system at $25^{\circ} \mathrm{C}$. 
Table 1. Temperature-dependent dielectric parameters for the binary mixture of 1-chloropropane and ethanol.

\begin{tabular}{|c|c|c|c|c|c|c|c|c|}
\hline \multirow{2}{*}{$\begin{array}{l}\text { Volume fraction of } \\
\text { 1-chloropropane }\end{array}$} & \multicolumn{2}{|c|}{$10^{\circ} \mathrm{C}$} & \multicolumn{2}{|c|}{$15^{\circ} \mathrm{C}$} & \multicolumn{2}{|c|}{$20^{\circ} \mathrm{C}$} & \multicolumn{2}{|c|}{$25^{\circ} \mathrm{C}$} \\
\hline & $\varepsilon_{0}$ & $\tau(\mathrm{ps})$ & $\varepsilon_{0}$ & $\tau(\mathrm{ps})$ & $\varepsilon_{0}$ & $\tau(\mathrm{ps})$ & $\varepsilon_{0}$ & $\tau(\mathrm{ps})$ \\
\hline 0.0 & $28.7(2)$ & $168.4(3)$ & 25.7 (1) & $158.5(1)$ & $25.1(1)$ & 146.1 & $24.3(2)$ & 133.9 (2) \\
\hline 0.1 & $24.1(3)$ & 138.9 (4) & 22.7 (2) & $125.6(2)$ & $22.2(3)$ & $102.7(3)$ & 21.9 (3) & $99.0(3)$ \\
\hline 0.2 & $23.3(6)$ & 75.5 (4) & $21.6(6)$ & $61.0(3)$ & $20.1(6)$ & $49.3(2)$ & $18.5(5)$ & 54.1 (3) \\
\hline 0.3 & $19.3(5)$ & $62.9(3)$ & 18.9 (4) & $58.6(3)$ & $18.2(4)$ & $42.8(2)$ & 17.0 & 48.9 (2) \\
\hline 0.4 & $18.9(4)$ & 49.9 (2) & $17.4(3)$ & $44.8(2)$ & $16.8(3)$ & 35.5 (1) & $14.8(3)$ & $44.2(2)$ \\
\hline 0.5 & $17.7(3)$ & $46.5(2)$ & $16.1(2)$ & 38.8 (1) & $15.8(2)$ & $34.2(1)$ & 13.9 (2) & $20.2(1)$ \\
\hline 0.6 & $16.3(1)$ & $30.8(1)$ & $14.4(1)$ & $25.8(1)$ & $13.3(1)$ & $22.6(9)$ & $12.6(1)$ & $16.8(7)$ \\
\hline 0.7 & $14.1(1)$ & $18.2(6)$ & $12.5(1)$ & $15.5(5)$ & $11.8(1)$ & $13.3(5)$ & $10.7(9)$ & $11.9(4)$ \\
\hline 0.8 & $12.0(6)$ & $11.5(3)$ & 10.9 (5) & $11.1(2)$ & $10.9(6)$ & $8.7(2)$ & $10.4(5)$ & $8.1(2)$ \\
\hline 0.9 & $11.6(1)$ & $9.8(9)$ & 10.7 (1) & 8.9 (7) & 9.9 (1) & $8.3(7)$ & $9.5(1)$ & $6.8(7)$ \\
\hline 1.0 & $11.2(1)$ & $7.7(8)$ & $9.0(6)$ & $6.1(3)$ & $8.9(5)$ & $5.8(2)$ & $8.3(4)$ & $5.7(2)$ \\
\hline
\end{tabular}

increase in the volume fraction of 1-chloropropane in ethanol. Decrease of dielectric constant is due to the transition of spherical molecular aggregates into elongated aggregates giving rise to parallel orientation of the dipoles. ${ }^{24}$ Shirke et al. drew similar conclusions for ethyl acetate-alcohol systems. ${ }^{25}$ For all the molecules under investigation, the relaxation time decreases with increase in the temperature from $10^{\circ} \mathrm{C}$ to $25^{\circ} \mathrm{C}$. This may possibly be due to that the increase in temperature causes alteration in molar volume, molecules to reorient with faster rate and increase in the rate of loss of energy due to large number of collisions. Analogous sorts of results are presented by Rewar and Bhatnagar. ${ }^{26}$ Other causes for the decrease in relaxation time are increase in the molar volume and the increase in the effective length of the dipole with increase in temperature. This is in agreement with other works in the literature. ${ }^{27}$

\subsection{Excess dielectric permittivity}

For dielectric properties of the mixture, the contribution of intermolecular bonds is studied in terms of the excess static dielectric permittivity. The excess dielectric permittivity can be written as ${ }^{17,28,29}$

$$
\varepsilon_{0}^{E}=\left(\varepsilon_{0}\right)_{M}-\left[\left(\varepsilon_{0}\right)_{E} X_{E}+\left(\varepsilon_{0}\right)_{C}\left(1-X_{E}\right)\right],
$$

where the subscripts $M, E$ and $C$ represents mixture, ethanol and 1-chloropropane, respectively, and $X_{E}$ represents the volume fraction of ethanol in mixture.

The plot of excess dielectric permittivity $\varepsilon_{0}^{E}$ of the mixture versus volume fraction of ethanol is shown in Fig. 2. The excess dielectric permittivity provides qualitative information about the multimer formation in the mixture as follows:

(i) $\varepsilon^{E}=0$ signifies that the liquids $E$ and $C$ do not interact at all.

(ii) $\varepsilon^{E}<0$ signifies that the liquids $E$ and $C$ interact in such a way that the total effective dipole gets reduced. The liquids $E$ and $C$ may form multimers leading to the less effective dipole.

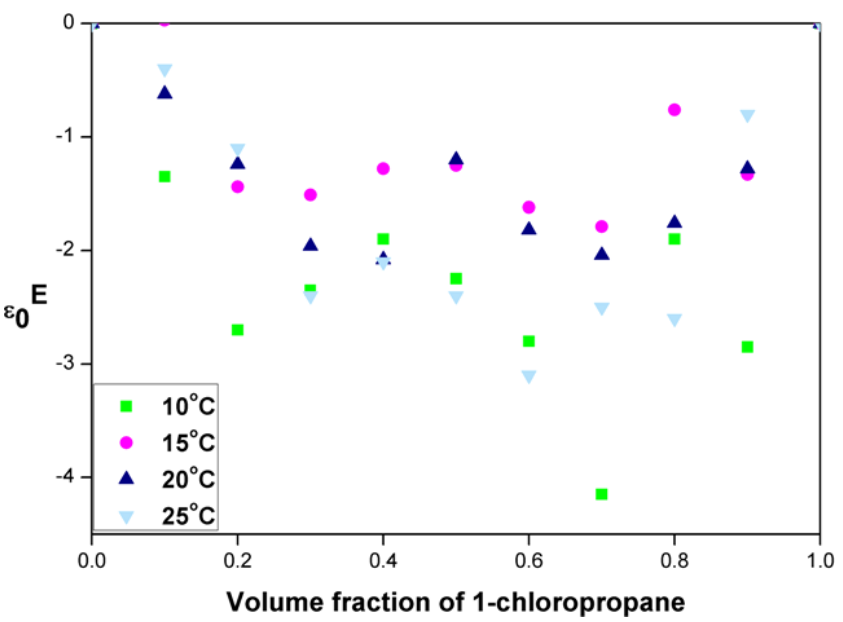

Fig. 2. Excess dielectric permittivities for 1-chloropropane-ethanol mixture from $10^{\circ} \mathrm{C}$ to $25^{\circ} \mathrm{C}$.

(iii) $\varepsilon^{E}>0$ signifies that the liquids $E$ and $C$ interact in such a way that the total effective dipole moment increases. There is a tendency to form multimers.

The peak value of minima for the excess permittivity at different temperatures is found at around $X_{E} \approx 0.7$. In this system, the negative values of the excess permittivity indicate weak hydrogen interface between ethanol and 1-chloropropane molecules. In addition, it points out that there is a multimer prototype.

\subsection{Excess inverse relaxation time}

The information related to dynamics of the solute-solvent interaction may be obtained by the excess properties related to the relaxation time in the mixture. The excess inverse relaxation time is defined as ${ }^{30,31}$

$$
\left(\frac{1}{\tau}\right)^{E}=\left(\frac{1}{\tau}\right)_{M}-\left[\left(\frac{1}{\tau}\right)_{E} X_{E}+\left(\frac{1}{\tau}\right)_{C}\left(1-X_{E}\right)\right],
$$




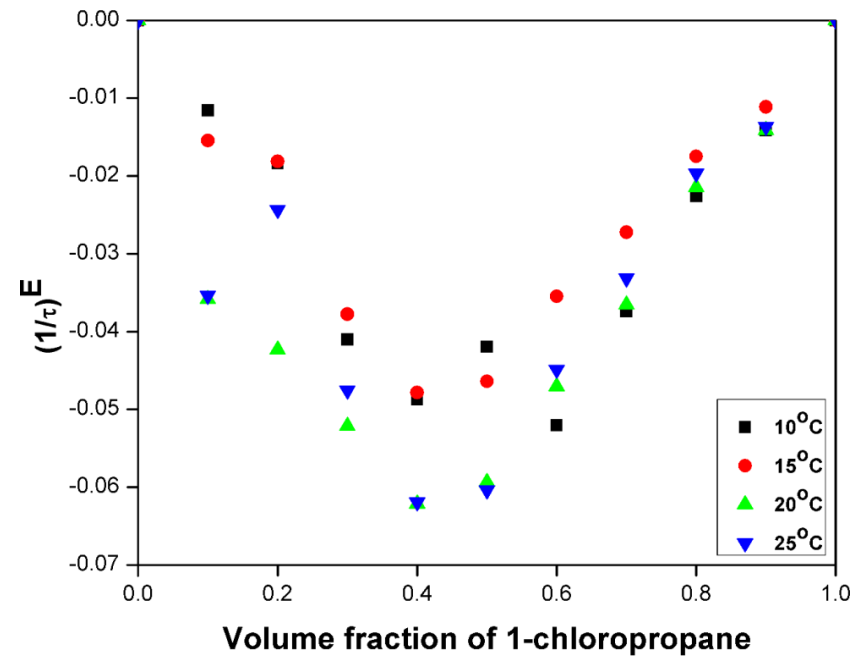

Fig. 3. Excess inverse relaxation times for 1-chloropropane-ethanol mixture from $10^{\circ} \mathrm{C}$ to $25^{\circ} \mathrm{C}$.

where $(1 / \tau)^{E}$ is the excess inverse relaxation time which represents the average broadening of dielectric spectra. $M, E$ and $C$ correspond to mixture, ethanol and 1-chloropropane, respectively. The information regarding the dynamics of solute-solvent interaction from this excess property is as follows:

(i) $(1 / \tau)^{E}=0$ : There is no change in the dynamics of $E$ and $C$ interaction.

(ii) $(1 / \tau)^{E}<0$ : The interaction of $E$ and $C$ produces a field such that the effective dipoles rotate slowly.

(iii) $(1 / \tau)^{E}>0$ : The interaction of $E$ and $C$ produces a field such that the effective dipoles rotate quickly, i.e., the field cooperate in the rotation of the dipoles.

The variations of excess inverse relaxation time $(1 / \tau)^{E}$ with volume fraction of 1-chloropropane in ethanol are shown in Fig. 3. The negative peak value of $(1 / \tau)^{E}$ has been found at around $X_{E}=0.4$. The value of $(1 / \tau)^{E}$ is less than zero, i.e., negative, for all the concentrations at all temperatures. This behavior suggests that there is slower rotation of the dipoles in the system.

\subsection{The Kirkwood correlation}

The structural information about the liquids from the dielectric relaxation parameter may be obtained by means of the Kirkwood correlation parameter $g$. This factor is also useful for obtaining information regarding orientation of electric dipoles in polar liquids. The $g$ for the pure liquid may be obtained using the following expression from Ref. 32:

$$
g \mu^{2} \frac{4 \pi N \rho}{9 K T M}=\frac{\left(\varepsilon \varepsilon_{0 m}-\varepsilon \varepsilon_{\infty m}\right)\left(2 \varepsilon \varepsilon_{0 m}+\varepsilon \varepsilon_{\infty m}\right)}{\varepsilon \varepsilon_{0 m}\left(\varepsilon \varepsilon_{\infty m}+2\right)^{2}},
$$

where $\mu$ is the dipole moment in the gas phase, $\rho$ is the density at temperature $T, M$ is the molecular weight, $K$ is the Boltzmann constant and $N$ is Avogadro's number; also $\varepsilon_{0}$ and $\varepsilon_{\infty}$ are the static dielectric constant and dielectric constant at high frequency and $g$ is the Kirkwood correlation factor for the $i$ th liquid system.

Modified form of the above equation can be used to estimate the Kirkwood correlation factor and to study the orientation of dipoles due to heterogeneous interaction in binary mixtures, which is expressed as follows:

$$
\begin{aligned}
\frac{4 \pi N}{9 K T} & {\left[\frac{\mu_{E}^{2} \rho_{E} V_{E}}{M_{E}}+\frac{\mu_{C}^{2} \rho_{C}\left(1-V_{E}\right)}{M_{C}}\right] \times g^{\text {eff }} } \\
& =\frac{\left(\varepsilon_{0 m}-\varepsilon_{\infty m}\right)\left(2 \varepsilon_{0 m}+\varepsilon_{\infty m}\right)}{\varepsilon_{0 m}\left(\varepsilon_{\infty m}+2\right)^{2}},
\end{aligned}
$$

where $g^{\text {eff }}$ is the Kirkwood effective correlation factor, $\mu_{E}$ is the dipole moment of ethanol, $\mu_{C}$ is the dipole moment of 1-chloropropane and $\rho_{E}$ and $\rho_{C}$ are the densities of ethanol and 1-chloropropane, respectively. $V_{E}$ represents the volume fraction of ethanol in 1-chloropropane. The value of $g^{\text {eff }}$ for ethanol is found to be 3.16 at $25^{\circ} \mathrm{C}$ which is greater than unity. This indicates the formation of H-bonded molecular multimers of binary mixture of 1-chloropropane and ethanol and favors parallel orientation.

It is also observed that $g^{\text {eff }}$ values vary with change in temperature, indicating that temperature change produces appreciable structural change. Determined values of Kirkwood correlation factor are plotted against volume fraction of 1-chloropropane, as shown in Fig. 4.

\subsection{Thermodynamic parameters}

The activation parameters namely enthalpy $\Delta H$ and entropy $\Delta S$ for dielectric relaxation process were calculated using the Eyring rate equation, ${ }^{33}$

$$
\tau=\frac{h}{K T} \exp \frac{\Delta H-T \Delta S}{R T},
$$

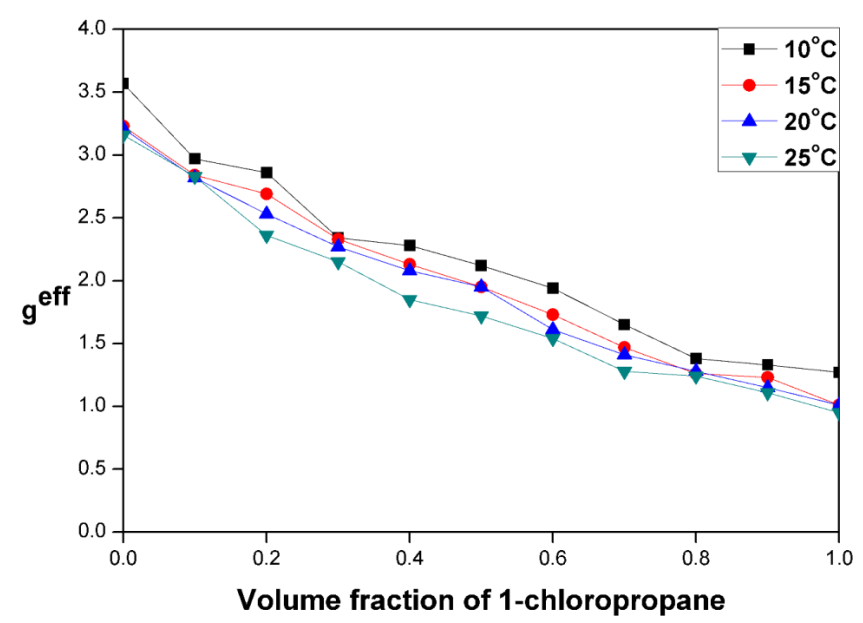

Fig. 4. Plot of Kirkwood effective correlation factor $g$ eff versus volume fraction for 1-chloropropane-ethanol mixture at the temperatures of $10-25^{\circ} \mathrm{C}$. 
Table 2. Thermodynamic parameters for the 1-chloropropane-ethanol binary mixture.

\begin{tabular}{lcc}
\hline $\begin{array}{l}\text { Volume fraction of } \\
\text {-chloropropane }\end{array}$ & $\begin{array}{c}\text { Enthalpy of activation } \\
\Delta H\left(\mathrm{~kJ} \cdot \mathrm{mol}^{-1}\right)\end{array}$ & $\begin{array}{c}\text { Entropy of activation } \\
\Delta S\left(\mathrm{~J} \cdot \mathrm{mol}^{-1} \cdot \mathrm{K}^{-1}\right)\end{array}$ \\
\hline 0.0 & $8.35(7)$ & $0.20(2)$ \\
0.1 & $14.6(2)$ & $0.22(1)$ \\
0.2 & $14.7(7)$ & $0.23(2)$ \\
0.3 & $12.6(8)$ & $0.22(2)$ \\
0.4 & $6.10(9)$ & $0.20(3)$ \\
0.5 & $34.2(9)$ & $0.30(3)$ \\
0.6 & $24.8(3)$ & $0.27(1)$ \\
0.7 & $17.6(9)$ & $0.25(3)$ \\
0.8 & $15.7(3)$ & $0.24(1)$ \\
0.9 & $13.8(2)$ & $0.24(9)$ \\
1.0 & $11.0(4)$ & $0.23(1)$ \\
\hline
\end{tabular}

where $\tau$ is relaxation time, $h$ is Planck's constant, $K$ is Boltzmann constant, $T$ is the temperature in $\mathrm{K}$ and $R$ is gas constant.

The molar activation enthalpy $\Delta H$ and entropy $\Delta S$ for pure and nine unusual concentrations were gauged and the values are reported in Table 2 . The positive values of activation enthalpy $\Delta H$ substantiate that heat is absorbed in the molecular reorientation process. The positive values of entropy indicate that activated system is less structured than the standard system. ${ }^{5}$

\section{Conclusion}

The complex permittivity spectra of 1-chloropropane-ethanol mixture are determined using TDR spectroscopy method in the frequency range of $10 \mathrm{MHz}-50 \mathrm{GHz}$. The dielectric relaxation parameters, i.e., static dielectric contact $\left(\varepsilon_{0}\right)$, relaxation time $(\tau)$, variation in excess permittivity, Kirkwood effective correlation factor ( $\left.g^{\text {eff }}\right)$, activation enthalpy $(\Delta H)$ and entropy $(\Delta S)$, have been reported for 1-chloropropaneethanol mixture for dissimilar temperatures. We summarize our findings as follows:

- The static dielectric constant of mixtures is found to be in the range of 24.3-8.3. In the 1-chloropropane-ethanol mixture, the dielectric permittivity decreases with increase in the concentration of 1-chloropropane.

- The relaxation time decreases with the addition of 1-chloropropane in ethanol, which may be due to the following reasons: (i) smaller size and fast rotation of 1-chloropropane molecules; and (ii) low viscosity of 1-chlorpropane.

- Kirkwood effective correlation factor $\left(g^{\text {eff }}\right)$ values are greater than unity, indicating the formation of $\mathrm{H}$-bonded molecular multimers of binary mixture of 1-chloropropane and ethanol and favoring parallel orientation.

- The positive values of enthalpy ensure endothermic reaction. The positive entropy of activation indicates that the activated state is more disordered.

\section{Acknowledgments}

The financial support from the Department of Science and Technology (DST), New Delhi, is gratefully acknowledged (Project No. DST PROJECT-SB/S2/LOP-032/2013). Ravindra V. Shinde is thankful to VNMKV, Parbhani, for sanctioning the study leave for research purpose. One of the co-authors Ashok C. Kumbharkhane is thankful to DST for providing financial assistance in the form of JRF.

\section{References}

${ }^{1}$ U.S. National Library of Medicine, Hazardous Substances Data Bank: Substance name: 1-chloropropane (2013), http://toxnet. nlm.nih.gov/cgibin/sis/search2/r?dbs+hsdb:@term+@DOCNO +5681 .

${ }^{2}$ G. D. Clayton and F. E. Clayton, Patty's Industrial Hygiene and Toxicology: Volume 2B: Toxicology, 3rd edn., Chapter 2 (John Wiley \& Sons, New York, 1981), p. 3525.

${ }^{3}$ FisherScientific, MSDS of 1-chloropropane (2018), http://fishersci.ca/viewmsds.do?catNo=AC109951000.

${ }^{4} \mathrm{G}$. Mamantov (ed.), Characterization of Solutes in Nonaqueous Solvents, Chapter 1 (Plenum Press, New York, 1976), p. 26.

${ }^{5}$ N. E. Hill et al., Dielectric Properties and Molecular Behavior (Van Nostrand Reinhold Company, London, 1969).

${ }^{6} \mathrm{~B}$. L. Clapp, The Chemistry of the OH Group, Chapter 1 (PrenticeHall, Englewood Cliffs, 1967), p. 19.

${ }^{7}$ G. I. Brown, Introduction to Organic Chemistry, 5th edn. (Orient Longman Pvt., Ltd., Calcutta, 1961).

${ }^{8}$ S. Mehrotra, A. Kumbharkhane and A. Chaudhari, Binary Polar Liquids: Structural and Dynamic Characterization Using Spectroscopic Methods, 1st edn. (Elsevier, Amsterdam, 2017).

${ }^{9}$ Y. Melendez-Pagan, B. E. Taylor and D. Ben-Amotz, Cavity formation and dipolar contribution to the Gauche-trans isomerization of 1-chloropropane and 1,2-dichloroethane, J. Phys. Chem. $B$ 105, 520 (2001).

${ }^{10}$ J. R. Durig, X. Zhu and S. Shen, Conformational and structural studies of 1-chloropropane and 1-bromopropane from temperature-dependent FT-IR spectra of rare gas solutions and ab initio calculations, J. Mol. Struct. 570, 1 (2001).

${ }^{11} \mathrm{~V}$. Sharma et al., Dielectric relaxation study of ethanol in benzene from microwave absorption data, Z. Nat.forsch. A 62, 406 (2007).

${ }^{12}$ A. D. Vyas and V. A. Rana, Dielectric relaxation of some rigid polar molecules and their binary mixture in benzene solution, Indian J. Pure Appl. Phys. 40, 69 (2002).

${ }^{13}$ V. S. Rangra and D. R. Sharma, Dielectric relaxation studies of binary mixtures of acetone and $N, N$-dimethylacetamide in the benzene solutions using microwave absorption data, Indian $J$. Pure Appl. Phys. 41, 630 (2003).

${ }^{14}$ A. Chaudhari, S. Ahire and S. C. Mehrotra, Dielectric relaxation study of pyridine-water and pyridine-sulfolane mixtures using time domain reflectometry, J. Mol. Liq. 94, 17 (2001).

${ }^{15} \mathrm{G}$. D. Rewar and D. Bhatnagar, Dielectric relaxation in ternary mixtures of benzotrifluorides, Indian J. Pure Appl. Phys. 40, 430 (2002).

${ }^{16}$ A. C. Kumbharkhane, S. M. Puranik and S. C. Mehrota, Dielectric relaxation of tert-butyl alcohol-water mixtures using a time-domain technique, J. Chem. Soc., Faraday Trans. 87, 1569 (1991). 
${ }^{17}$ S. C. Kumbharkhane et al., Dielectric relaxation study of hexamethylphosphoramide-water mixtures using time domain reflectometry, J. Chem. Phys. 99, 2405 (1993).

${ }^{18}$ J. B. Hasted, Aqueous Dielectrics (Chapman and Hall, London, 1973).

${ }^{19}$ R. H. Cole et al., Time domain reflection methods for dielectric measurements to $10 \mathrm{GHz}$, J. Appl. Phys. 66, 793 (1989).

${ }^{20}$ S. Harviliak and S. Negami, A complex plane analysis of $\alpha$-dispersions in some polymer systems, J. Polym. Sci. 14, 99 (1996).

${ }^{21}$ K. Udo, Microwave dielectric properties of liquids, Radiat. Phys. Chem. 45, 549 (1995).

${ }^{22}$ K. S. Cole and R. H. Cole, Dispersion and absorption in dielectrics 1: Alternating current characteristics, J. Chem. Phys. 9, 341 (1941).

${ }^{23}$ D. W. Davidson and R. H. Cole, Dielectric relaxation in glycerin, J. Chem. Phys. 18, 1417 (1950).

${ }^{24} \mathrm{P}$. Sivagurunathan et al., Dielectric study of butyl methacrylatealcohol mixtures by time-domain reflectometry, Physica B 387, 203 (2007).

${ }^{25}$ R. M. Shirke et al., Temperature dependent dielectric relaxation study of ethyl acetate-alcohol mixtures using time domain technique, J. Mol. Liq. 94, 27 (2001).

${ }^{26}$ G. D. Rewar and D. Bhatnagar, Microwave absorption and relaxation processes of ternary mixtures of non-rigid polar liquids, Indian J. Pure Appl. Phys. 39, 707 (2001).
${ }^{27}$ P. J. Singh and K. S. Sharma, Viscoelastic and dielectric relaxation studies of some ketones in dilute solutions of benzene, paraffin oil and their mixtures, Indian J. Pure Appl. Phys. 34, 388 (1996).

${ }^{28}$ A. C. Kumbharkhane, S. M. Puranik and S. C. Mehrotra, Temperature dependent dielectric relaxation study of ethylene glycolwater mixtures, J. Solut. Chem. 21, 201 (1992).

${ }^{29}$ R. J. Sengwa, V. Khatri and V. Sankhala, Dielectric behaviour and hydrogen bond molecular interaction study of formamide-dipolar solvents binary mixtures, J. Mol. Liq. 144, 89 (2009).

${ }^{30}$ Z. Fakhar, A. Ghanadzadeh and B. Honarparvar, Dielectric study of molecular association of polar-polar binary mixtures (benzyl alcohol + aliphatic alcohols) at 298.2 K, J. Iran. Chem. Soc. 12, 1363 (2015).

${ }^{31}$ A. Chaudhari et al., Temperature dependent dielectric relaxation study of aniline in dimethylsulphoxide and dimethylformamide using time domain technique, J. Korean Chem. Soc. 45, 201 (2001).

${ }^{32}$ J. G. Kirkwood, The dielectric polarization of polar liquids, J. Chem. Phys. 7, 911 (1939).

${ }^{33}$ S. Glasstone, K. J. Laidler and H. Eyring, Theory of Rate Processes (McGraw-Hill, New York, 1941). 